\title{
Enhancing appropriate environmental design in healthcare facilities for the inhibition of nosocomial infection
}

\author{
Khai Tran $\operatorname{Van}^{1 *}$ \\ ${ }^{1}$ Hong Bang International University, 215 Dien Bien Phu,, Bình thanh District, Ho Chi Minh City, \\ Vietnam
}

\begin{abstract}
A Nosocomial infection also known as a hospital-acquired infection (HAI), is an infection that is acquired in a hospital or other health care facility are increasing even in Contemporary Hospitals of Vietnam. This study described how HALs occur in patients during the time under medical care in healthcare facilities as during hospitalization, the patient is exposed to pathogens through different sources which mostly are environment, healthcare staff, and other infected patients. The prevention and control of HALs requires the implementation of infection control interventions. The role of infection control mentioned in this study is to review and approve construction and architecture design measures to ensure they meet the demands for minimizing nosocomial infections. This study examines how HALs spread among hospitalized patients via environmental routes and how the application of appropriate design of the hospital plays a critical part in preventing the spread of infection. As the application of new technologies has application range within stamina in cases of climate changes, the findings of this study is the adoption of flexible transformable hospital design strategies in Vietnam to prevent infection.
\end{abstract}

\section{Introduction}

In current times of Vietnam, some hospitals have been pioneers in the application of new technologies. Besides medical care efforts, many technological system including architectural spaces such as isolation rooms, hand wash facilities, Hospital ventilation system was paid attention to. But many cases shown that if unexpected factors appear in nature, that technological systems may fail. Therefore, the research aims to form some of the effective architectural planning with appropriate flexible design of sustainable models for hospital buildings in the recent climate change context.

The study utilised an exploratory, sequential, mixed methods design to allow for the development of a survey designed to collect data on outbreaks of HAIs and the implementation of infection control interventions in Hospitals of Vietnam.

Based on the reviews on the impacts of climate changes to infectious diseases with modes of transmission, this study aims to show that the application of advanced theories of sustainability architecture in a warm climate context are necessary for healthcare facilities.

\footnotetext{
*Corresponding author: dduonghai2003@gmail.com
} 
Friendly environmental solutions provided alongside advanced technological systems will help prevent airborne infections such as natural light is also a good germicidal factor. Therefore the application of adaptive transformable hospital architectural design appropriate to the context of the country is an innovative attitude of the current times

Theories on prevention and control of HALs were analysed through evidence based surveys in order to answer the research questions of identifying the effective architectural and construction appropriate solution for the inhibition of HAIs. Furthermore, the application advanced solutions such as planning with clear hospital functional zonings, incorporating the flexibility and adaptive architecture design by modular solutions, simplified installation with system integration could meet the change of functional demands and the innovations of healthcare facilities.

\section{Literature review}

\subsection{Review on the impact of climate changes on the rise of infectious diseases}

The average temperature of Vietnam, a country located in the Asia-Pacific region ranges from $21^{\circ} \mathrm{C}$ to $27^{\circ} \mathrm{C}$, and increases from the North to the South. The average annual rainfall is from $1,500-2,000 \mathrm{~mm}$ and humidity is around $80 \%$. As the impact of complex terrain, monsoon operation and the sea, Vietnam usually suffers extreme weather phenomena such as hurricanes, floods. Studies claimed that the warm climate is favourable for the existence of infectious diseases.

The most general definition of climate change: is a change in the statistical properties of the climate system when considered over long periods of time. The term climate change has become synonymous with anthropogenic global warming [8] particularly the rise in temperature is related to infectious diseases.

McMichael A.J, Haines .A, Slooff .R, Kovats. S [12] cited that a warming and unstable climate is playing an ever-increasing role in driving the global emergence, resurgence and redistribution of infectious diseases, while climate temperature affects their growth and survival.

Zoologist Daniel Brooks [4] said: "There is an enormous possibility of diseases passing to new hosts...It's going to intensify as climate change progresses." Climate change plays an important role which affects infectious disease occurrence which leads to an apparent increase in many infectious diseases, particularly some newly-circulating ones which vary greatly in their mode of transmission and type including the viruses, bacteria, protozoa and multicellular parasites. Changes in infectious disease transmission patterns are a likely major consequence of climate change.Cimons Marlene [5] agrued that other infectious diseases, such as salmonellosis cholera and giardiasis, may show increased outbreaks due to elevated temperature and flooding .

Many researches shown that climate change helps cholera and salmonella outbreaks. [6] The dependance on technological measures will have risks when the mechanical ventilation systems in some cases may be inept and increase the transport and dissemination of infectious agents.

Recent scientific researches has shown that climate changes may spark a whole host of similar, global epidemics recently spread into unexpected places, as climactic fluctuations have pushed species such as Ebola and the West Nile virus into new environments. So climate change particularly global warming is a factor that in many cases could help viruses expand their range and make a comeback. 
Climate Change in Southeast Asia By 2080, sea levels are expected to rise by as much as $40 \mathrm{~cm} .2$ [22 ] East and Southeast Asia large cities including Bangkok, Manila, Ho Chi Minh City and Jakarta are at risk as sea levels rise. The epidemicity of disease vectors, such as mosquitoes and sand flies are highly climate sensitive so alterations to the geographical and seasonal distribution of diseases they transmit could change considerably. Malaria, dengue, Zika, and Japanese B encephalitis are among some of the more common infectious diseases of Asia likely to be affected [22].

\subsection{Review of the modes of infection transmission}

Many researches had assumed the modes of infection transmission in hospitals. Most of the studies agreed that Microorganisms are transmitted in health care facilities by the following main routes: Contact ; Droplet ; Airborne ; Common vehicle, which must be examined to prevent the risk of transmission of infectious disease to new hosts.

\subsubsection{Contact transmissions}

Contact is the most common mode of transmission of infection in hospitals which may be subdivided into direct contact, indirect contact and contact with droplets.

Direct contact: Infectious diseases are commonly transmitted through direct person-toperson contact. Transmission occurs when an infected person touches or exchanges body fluids with someone else. [25]. Direct contact refers to person-to-person spread of microorganisms through physical contact between the infectious agent including the contaminated hands or gloves of health care worker with the skin or mucous membranes of the recipient. Hand washing by the medical staff members is one of the ways to prevent transmission by the contact route.

Indirect contact: Indirect contact occurs when a susceptible person comes in contact with a contaminated object. Examples include door knobs, keyboards, fabrics where patients have open wounds, invasive devices contacted. Specific detailing design for easy cleaning, disinfection, and sterilization of hospital objects are essential to prevent nosocomial infection acquired from contaminated items and equipment.

Contact with droplet transmission: A person with a droplet-spread released infected secretions that spread through the air to the oral or nasal mucous membranes of a person nearby. Microbes in droplet nuclei (mucus droplets) can travel up to about 1 meter. The droplets don't remain suspended a long time in the air but settle on surfaces. Surfaces of materials of architectural elements such as partitions must be solid and smooth enough to be able to prevent the suspension of droplets.

\subsubsection{Airborne transmission}

The transmission of pathogens through the air has been noticed a long time ago, but not been solved effectively. Damani N. N. assume that The attendant microorganisms can proliferate in various indoor ecological niches and, if subsequently disbursed into the air, serve as a source for airborne healthcare-associated infections [8].

Airborne Transmission of disease caused by dissemination of droplet nuclei that remain infectious when suspended in air over long distance $(>1 \mathrm{~m})$ and time [29]. Further on, airborne transmission occurs when fine microbial particles or dust particles containing pathogens remain suspended in the air for a prolonged period, then are spread widely by air currents and inhaled which may cause infection when a susceptible person inhaling the infectious air flow and dusts. A variety of airborne infections in susceptible hosts can result from exposure to clinically significant microorganisms released into the air when environmental reservoirs (i.e., soil, water, dust, and decaying organic matter) are disturbed particularly in the demolishing of buildings and brought indoors into a healthcare facility by 
people, air currents, water, construction materials, and equipment [8]. The construction dust discharged from demolishing buildings is very harmful..

"Airborne transmission refers to infectious agents that are spread via droplet nuclei (residue from evaporated droplets) containing infective microorganisms. These organisms can survive outside the body and remain suspended in the air for long periods of time. They infect others via the upper and lower respiratory tracts." [8] Some infectious agents can travel long distances and remain suspended in the air for an extended period of time. [10]. Diseases that are commonly spread by coughing or sneezing include bacterial meningitis, chickenpox, common cold, influenza, mumps, strep throat, tuberculosis, measles, rubella, whooping cough, leprosy and SARS (Severe acute respiratory syndrome) .

Thus the physical design of some hospitals have been pioneers in the application of new technologies such as the service system of advanced techniques, especially the Heating, Ventilation and Air Conditioning system (HVAC). Having sterile filtration is practical. However, placing complete faith in this system is not only a fashionable mode but a bit less of enlightenment on the trend of sustainable architecture era. Each technological system has application range within stamina. If unexpected factors appear in nature, that technological system may become a double-edged sword.

\subsubsection{Droplet trasmission}

"Droplet transmission occurs when respiratory droplets generated via coughing, sneezing or talking contact susceptible mucosal surfaces, such as the eyes, nose or mouth. Transmission may also occur indirectly via contact with contaminated formites with hands and then mucosal surfaces. Respiratory droplets are large and are not able to remain suspended in the air thus they are usually dispersed over short distances."[7] Since droplets fall to the ground within a few feet, this type of transmission requires close proximity

The pathogen-containing particles, also called Flügge droplets (after Carl Flügge), are $0,1-2 \mathrm{~mm}$ in diameter, and are reduced by evaporation to droplet nuclei - small (smaller than $100 \mu$ in diameter), dry particles that can remain airborne for long periods

Organisms spread by droplet transmission include respiratory viruses (e.g., influenza, parainfluenza virus, adenovirus, respiratory syncytial virus, human metapneumovirus), Bordetella pertussis, pneumococci, diphtheria, and rubella [12].

Table 1: Numbers of Disability-Adjusted life-years Due to causes that are attributable to climate change. (Source: WHO.)

\begin{tabular}{|lcc|}
\hline $\begin{array}{c}\text { Numbers of Disability-Adjusted Life-Years Due to Causes That Are Attributable } \\
\text { to Climate Change, as of 2000.* }\end{array}$ & $\begin{array}{c}\text { DALYs/Million } \\
\text { Population }\end{array}$ \\
Region & Total DALYs & 3071.5 \\
Africa & $1,894,000$ & 1586.5 \\
Eastern Mediterranean & 768,000 & 188.5 \\
Latin America and Caribbean & 121,000 & 1703.5 \\
Southeast Asia & $2,572,000$ & 111.4 \\
Western Pacific & 169,000 & 8.9 \\
\hline Developed countries & 8,000 & \\
\hline
\end{tabular}




\subsubsection{Waterborne transmission}

Hospital water is a source of infectious microorganisms when hospital buildings draw the infected water from the municipal water supply. Corrosion damaged distribution pipelines, storage tank, reservoirs, poor water system design and water stagnation are also other transmission factors. Examples of common waterborne pathogens bacteria found in potable water include Legionella pneu - mophila, Stenotrophomonas maltophilia, Aeromonas spp., Acinetobacter spp., Enterobacter spp., Flavobacterium spp. [8].

\subsubsection{Vector-borne.}

A vector is an organism that does not cause disease itself but that transmits infection by conveying pathogens from one host to another [15]. Vectors may be mechanical or biological. A mechanical vector picks up an infectious agent on the outside of its body and transmits it in a passive manner.

\subsection{Review on concepts of inovative Architecture Design methodologies related with the cases of HAls}

\section{Appropriate Architecture Design}

The spectrum of sustainable architecture consists of active involvement of the occupants into micro-climate control within the building, and the natural environment as the physical context [6]. Sustainable architecture may relate to or include the concepts of appropriate, adaptive and evolutionary architecture .

Jean-Nicolas-Luis Durand an architect, professor, and theorist from a French Architectural School of Public Works cited : For a building to be appropriate, it must be solid, healthy, and comfortable. But the current problem is many architectural hospital design projects are unaware of the serious relations between climate change and emerging infectious diseases. It was broadly understood that the sustainable architectural design in warm climate countries should focus on the micro-climate control within the building, including the control of of transmission of infectious disease in the environment of healthcare facilities.

\subsubsection{Flexible architecture}

Robert Kronenburg [13] investigated the genre of flexible architecture - buildings that are intended to respond to changing situations in their use, operation, or location and defined that: This is architecture that adapts rather than stagnates; responds to change rather than rejects it; is motive rather than stati. [12].

\subsubsection{Adaptive modelling}

Adaptive modelling in entropy evolution is a design alternative for sustainable architecture. [8] The aim of an evolutionary architecture is to achieve in the built environment the symbiotic behaviour and metabolic balance found in the natural environment [1].

\subsubsection{Evolutionary architecture}

An evolutionary architecture supports guided, incremental change on both quality and quantity of elements as a first principle across multiple dimensions. Evolutionary architecture design incorporates time as an important resource.

\subsection{The application of Medical computerized information system}

The construction of medical computerized information is important to improve the hospital medical care. The establishment and use of these information systems played an important 
role in improving the degree of patient satisfaction, enhancing hospital efficiency and healthcare quality, protecting the safety of healthcare, and reducing healthcare costs. They display laboratory test results, patient allergies, lists of medications the patient is taking, medical and nursing diagnoses, patient demographics, and providers' notes. IS EHR systems also electronically transmit test results from laboratories, radiology centers, and other testing facilities to clinicians quickly and efficiently. Many systems allow clinicians to submit computerized medication orders and care instructions to pharmacies and other providers [17].

Computerized physician order entry and CDS are regarded as one of the most beneficial health information technologies for improving patient safety. In addition, ADC systems and PDMS seem to improve patient safety in critical care setting.

\section{$3 \quad$ Materials and method}

\subsection{Materials}

The materials in this study contained statistics from the health ministry commented that on average the rate of HAIs in Việt Nam is 7 per cent and one of the leading threats to patients' lives. Deputy Minister Nguyễn Viết Tiến once said that HAIs had a large impact on patients' treatment process. Many cases were hospitalised when their conditions were not critical, but worsened due to hospital's infection control. Tiến cited that poor infection control contributed to a measles outbreak in April 2014, killing more than 110 children and hospitalising thousands nationwide. It also played a role in the deaths of four pre-term and low birth weight infants at Bắc Ninh Hospital of Obstetrics and Pediatrics in November 2017.

A study titled "Burden of Hospital Acquired Infections and Antimicrobial use in Vietnamese Adult Intensive Care Units" by Dr Vũ Đình Phú from the National Hospital of Tropical Diseases and colleagues in 2016, revealed that the HAI prevalence in 15 Intensive Care Units of hospitals in the country ranged from 19.3 per cent to 31.1 per cent. Another study, which was carried out from 2012-13 on hospital sizes ranging from 280 to 2,362 beds, with participating ICU sizes ranging from 10 to 60 beds, found that 29.5 per cent of patients staying in ICUs had at least one HAI [11].

Tạ Thị Hằng Nga, head of the Infection Control Department of Đống Đa Hospital, cited that the hospital environment, medical materials and health staff might all be threats of infection, and she further on cited: "Infections from the hands of health staff are mostly threatening if they do not properly wash their hands" [10].

Statitics from researches in Asia and West Africa shown that fewer of the studies on weakness of infection prevent control focused on the shortcoming of hospital design or ventilation solutions rather than the lack of equipment. 


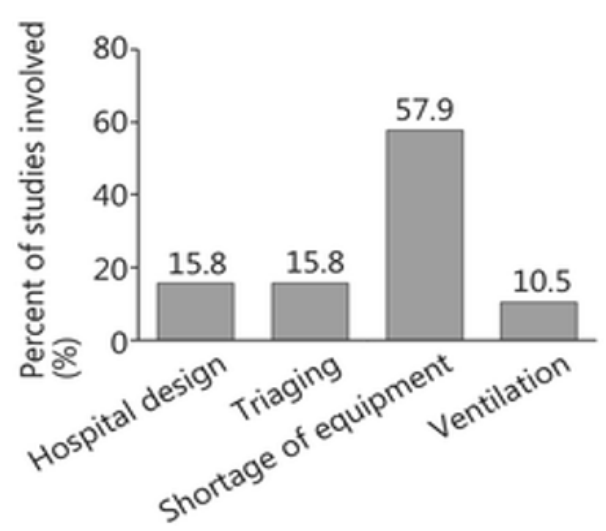

Fig. 1. Percent of studies involved in certain topics of on causes of healthcare infection transmission. (Source:[18])

\subsection{Researh Method}

This study applied the method of comprehensive analysis and synthetic for most of the ploblems and data stated.

The contents of literature reviews of this study were analysed through descriptive statistics in order to answer the research questions and the full hypothesis was then tested by regression analysis estimating the relationships between a dependent variable and one or more independent variables

As the situation in a hospital differs from that in other types of healthcare facilities in a number of ways and some study of. the results show only benefit in a single center, such as the actual situation of inpatients who are at high risk for acquiring infections, this study offered insight for continuing research which should focus on the reason of the selection as well as the development of preventive measures applied in the healthcare facilities..

The object of this publication is not to provide detailed technical schedules for the performance of individual measures but to discuss the use of the more important design strategies, indicate when and where should the solutions be applied, and show how the change of the architectural environment should be integrated with the rest of the hospital's infection control activities.

\section{$4 \quad$ Results}

\subsection{Incorporate appropriate architecture design and advanced construction technologies optimiizing hospital infection prevention possibilities}

A comprehensive planning and design solution is possible for the integration of multiple infrastructure systems, accommodate appropriate and future technologies with regulatory changes including the infection prevention measures to optimize building performance.

\subsection{Achive the flexibility of adaptive architecture by modular solutions, simplified installation, and system integration}

The flexibility by modular solutions, simplified installation, and system integration in architecture design could easily meet the change in space and the innovations of hospital facilities. Most hospital buildings are routinely used for 50 years or more - but at the same 
time individual rooms may be changed or replaced after as few as seven years, as clinical methods and equipment change to improves hospital performance [12]. In this way, the installation of new isolation rooms to prevent the transmission of pathogens through the air can be more easily done.

The flexibility of the structural system must develop in many directions and many locations on the same time create conditions for the development of interior space: flexibility in allowance of use function, flexibility in disposal flexibility, flexibility in the arrangement of space, shown in the ability of extending building blocks or connecting with urban infrastructure systems. The interactions amongst all the parameters compose a complex system of sustainable architecture design, of which the conventional linear and fragmented design technologies are insufficient to indicate holistic and ongoing environmental performance [9].

R.Sprow claimed that a frequently used planning module that fits these criteria is a bay size of $9.2 \mathrm{~m} \times 9.2 \mathrm{~m}$ which neatly fits a cluster of 6 exam rooms with a $1.6 \mathrm{~m}$ corridor, or two patient rooms with a nominal width of $4 \mathrm{~m}$, or a group of 6 parking spaces, this size module also is within the capacity of a minimum depth flat slab concrete structure or a simple steel structure, without long spans [19]. Experiments through practice in Viet Nam identified that a bay size of $8.4 \mathrm{~m} \times 8.4 \mathrm{~m}$ meeting the demands of load bearing was linked to marketing responsiveness.

The application of adaptive, flexible architecture can help reduce the demand for demolishing obsolete hospital buildings thus reduce the discharge of construction dust.

\subsection{Planning with clear hospital functional zonings.}

Planning that is clear and modular can help the replacement of functions of whole architecture blocks. This planning solution enables the flexibility in changes of technological system, whether advanced or appropriate to meet the demands of infection prevention.

Clear functional zonings or composition of spaces must be done so that the traffic flow can be easily modified in accordance with the new selected technological system. Old systems are being replaced by electronic kiosks, computerized direction systems with more interactive systems. Therefore the traffic flow of all kinds of different patients and staff does not overlap and reduce the intensity of travelling, thus minimizing the possibities of infection contact transmission. The choice of proper entry to connect from the urban infrastructure system is also important, particularly the main entrance, the entrance to the outpatient clinic and the emergency department must be visible for all, particularly patients.

Special attention must be paid to zones such as the ICU and the surgical zone that must be isolated from the main traffic routes, in particular the axis vertical traffic such as elevators shafts and staircases in order to reduce travelling intensity and avoid infected airflow from stack effect to minimize the effect of airborne transmission. A good example is the surgery zone in Cho Ray hospital which was located a distance away from the main elevator lobby.

\subsection{Enhance the application of Electronic medical records}

Electronic medical records (EMR) started as a way to eliminate the time and errors that came with the manual charting of patient data. Peviously the patient information was only able to be viewed within one office so, if a patient were transferred to another hospital, their medical information would not follow adequately. Electronic health records were created to allow the sharing of patient data throughout different healthcare facilities. With EMR or EHR software, a patient who is moved to another medical treatment facility can be properly 
treated because different medical staff members are able to access their information. . Further on, EMR also reduce the demands of physicans travelling around to many spaces thus eliminate the direct transmission of infectious diseases.

\subsection{Adaptive and evolutionary architecture must give priority to the application of natural factors as environmental control measures for airborne infectious deseases}

Long ago, the famous nurse Florence Nightingale, the first person to launch the hospital ward model, stated that natural daylight and fresh air are effective elements to sterilize and reduce the infection phenomenon in hospitals. Advanced research and experiments have shown the effective solutions: Friendly environmental solutions should be provided alongside advanced technological systems as natural light is also a good germicidal factor to help prevent airborne infections

\subsection{Enhance natural ventilation to reduce the intensity of airborne infection sources}

To help prevent airborne infections, adequate ventilation in healthcare facilities in main hospital spaces are necessary. When natural ventilation alone cannot satisfy the recommended ventilation requirements, alternative ventilation systems, such as hybrid (mixed-mode) natural ventilation should be considered, and then if that is not enough, mechanical ventilation should be used [2]. For natural ventilation, the following minimum hourly averaged ventilation rates should be provided as:

- $160 \mathrm{l} / \mathrm{s} /$ patient (hourly average ventilation rate) for airborne precaution rooms (with a minimum of $80 \mathrm{l} / \mathrm{s} /$ patient) (note that this only applies to new health-care facilities and major renovations);

$-60 \mathrm{l} / \mathrm{s} /$ patient for general wards and outpatient departments;

$-2.5 \mathrm{l} / \mathrm{s} / \mathrm{m}$ for corridors and other transient spaces without a fixed number of patients.

Table 2: Estimated air changes per hour and ventilation rate for a $7 \mathrm{~m} \times 6 \mathrm{~m} \times 3 \mathrm{~m}$ patient room in a ward (Source [2]).

\begin{tabular}{lcr}
\hline Openings & ACH & Ventilation rate $(1 / \mathbf{s})$ \\
\hline Open window $(100 \%)+$ open door & 37 & 1300 \\
Open window $(50 \%)+$ open door & 28 & 975 \\
Open window $(100 \%)+$ closed door & 4.2 & 150 \\
\hline
\end{tabular}

The SARS (Severe acute respiratory syndrome) outbreak of 2003 which started in southern China caused an eventual 8,098 cases, resulting in 774 deaths reported in 37 countries. On this SARs outbreak, in Vietnam, the lives of many patients and healthcare workers in an International hospital were lost when relying on mechanical ventilation, but no lives were lost in a nearby local hospital that is the National Tropical disease Hospital using natural ventilation and sunlight 


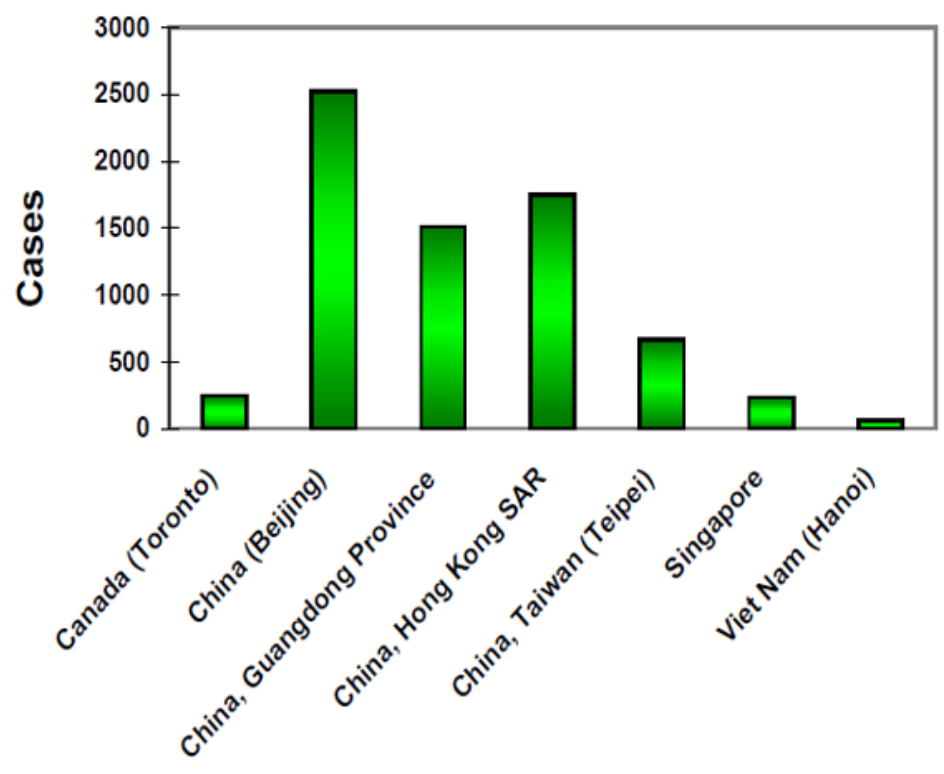

Selected sites

Fig.2. Probable SARs cases in selected areas of Hanoi(Source: Concensus Document on the Epidemiology of SARS WHO/CDS/CSR/GAR/2003).

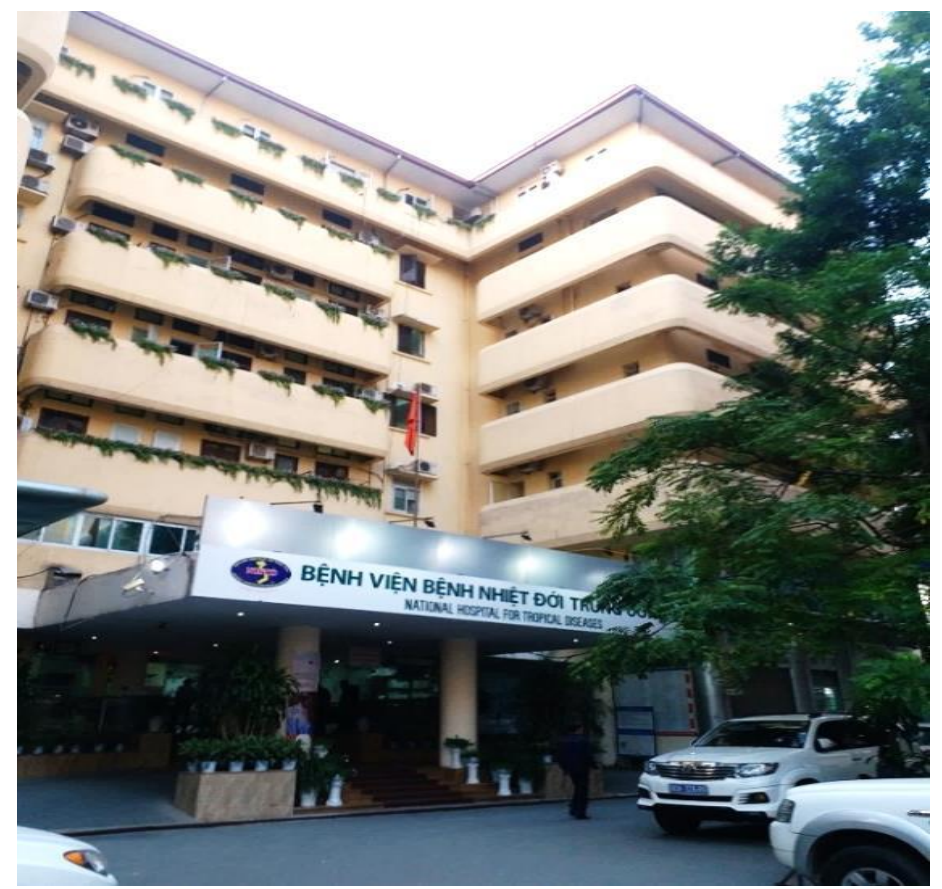

Fig.3. National Tropical desease Hospital in direct natural sunlight and ventilation (Source: BAOMOI.COM) 


\subsection{Enhance the Sunlight - nature's disinfectant by flexible building envelope elements of rooms and corridors.}

Experiments undertaken in the USA and the UK between 1941 and 1944 demonstrated the extraordinary and remarkable effectiveness of daylight in killing the bacteria streptococci [3]. The blue light in skylight was found to be particularly effective. Trials also examined the bactericidal effects of artificial light, which was found to have little value as a disinfecting agent.

Even diffused daylight passing through two layers of glass from a north window was found to be highly effective in killing haemolytic streptococci within 13 days, with the same strain surviving in the dark, at room temperature, for 195 days [19]. Furthermore, patients assigned to rooms with an open view of a natural setting had shorter postoperative stays than rooms looked onto a solid wall.

The pathogen usually is transmitted by air focus significantly at middle corridors of inpatient wards. Therefore layout of wards with side corridors are favourable. If this cannot be applied due to economical considerations, then some parts of the corridor should be openable. Wards with pavillion types should not be closed at the ends to ease ventilation or natural lighting. This design pattern will allow extension works when necessary as following the Evolutionary Architecture idea.

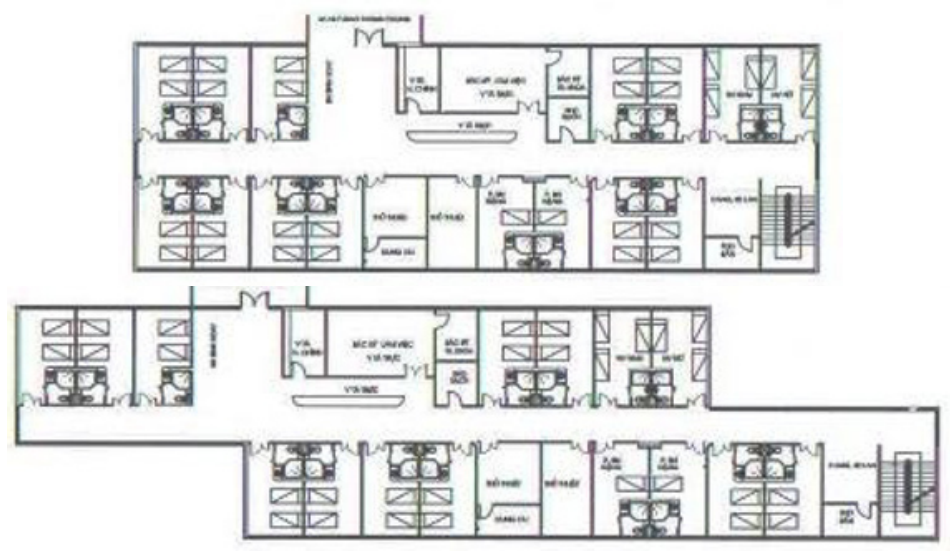

Fig.4. Designed layout of Hospital Wards with open ends or side corridors (Source: Tran Van Khai)

The diagnosis and treatment divisions with high requirements of sterilization should have an isolation room at the entrance. Therefore isolation rooms before the entrance to the areas requiring high sterilization using high air pressure in order to avoid infected air coming in must be flexible in the addition installation. The substitution of automatic door handles at any places must be considered.

In order to minimize the direct transmission phenomenon, it is necessary to install sinks at many places which are easily identifiable and accessible. The installation of handwashing basins in hospitals is one of the ways to prevent transmission by the contact route. Nowadays, it is possible to reach the criteria of 1 washbasin/4 patients. A nurse can come into contact with patients more than 100 times per day, if there is not an accessible hand basin, this would facilitate for disease transmission. These sinks do not coincide with the sinks in the restroom of the patient rooms in the previous plans or arranged in front of the bathroom doors. The partitions or walls on the ward corridors must be designed to have separate sinks fixed on when necessary, outside any expected patient rooms. The arrangement of sinks with hand sanitizer in front of the doors of every patient room also 
contributes to prevent the indirect transmission modes such as via keyboard, doorknobs and so on.

\subsection{Application of advanced adaptive finishing materials and construction technologies solutions in hospital buildings.}

It was also Florence Nightingale who stated that the plaster used in construction, which has many tiny voids, was thought to be the locality and transmission of pathogenic factors. So it is necessary to choose the type of plaster with high solidity or to use covering material such as special paints.

The ceilings mostly should be the type of large plate. In particular, the ceiling of the operating room and some other areas must be made by concrete placed monolithically, fixed by hidden light bulbs. When replacing broken light bulbs the workers shall come from above the ceiling.

In the case of designing the flexibility of a hospital building, the building components such as the structural columns and beams, floor slabs, roof and foundations are fixed, but the partitions could be movable. Even part of the building structure may be designed to change its characteristics or expand, particularly the components on the external envelop which are in accordance with the changes of operating functions and facilities of the hospital.

\subsection{Create a healthy, friendly environment to achive the sustainability features}

Lastly, although not directy concerned with the infection prevention requirements, it is necessary to enhance natural day light and natural ventilation to create a healthy, friendly environment for people and nature by adaptive expandable architecture measures.

In addition, create safe psychology for patients on vision and sound, clearly show the entrance, avoid corridors that are too long and avoid seeing unfavorable views.

Create spaces to install facilities as beds for relatives of patients in the patient rooms under the direction of doctors, do not misunderstand that modern hospitals are intolerant to the presence or care of relatives. Enhance the ability to facilitate for the treatment medical staff such as: select good places contacted directly with nature for station of nurses on duty, and have an internal communication system.

Intergrating large monitor screens into the hospital spaces makes it easy to put vital information into contextually appropriate locations as integrating information technology in the hospital now is an ultimate measure of this operational point of view.

\section{Enhance the application of Medical computerized information system in the environment of healthcare facilities}

Computerized physician order entry (CPOE) allows physicians to place lab and imaging orders, prescriptions and other notices electronically, reducing the error of hand-written orders and allowing the patient's other physicians within the EHR network access to the order. That means, if a patient is prescribed a drug from his/her cardiologist and they are on the same EHR, the primary care physician will have access to the prescribing information.

EHRs are much better organized than paper charts, when allowing for faster retrieval of diagnostic, lab or imaging results. It is also likely that an EHR will have an electronic problem summary list that outlines a patient's major illnesses, surgeries, allergies and medications in the past and present times. This not only reduces efforts, time, but also dramatically reduces errors such as duplicate prescriptions or drug interactions and potential harm to the patient There is substantial evidence that implementing an electronic 
medical record reduces medical errors and improves patient's safety [16]. Doctors and physician will also reduce the efforts of travelling around to many places particularly the diagnostic rooms and will limit the opportunity of HAIs through direct or indirect contact.

Architects must design spaces for the installation of electronic devices such as the sever room and cables.

\section{Discussion}

When developing the quote: "A house is a machine for living in" of Le Corbusier, we can accept the dominance of the perspective: A hospital is not only a house but also a machine.

Flexible architecture is a design form that is by its essence crossdisciplinary and multifunctional and consequently, is frequently innovative and expressive of contemporary design issues [13] Attention must be strictly paid to the flexibility adaptation of the elements of the surgery or ICU zone. Removable panel with advanced materials must be used for many partitions rather than brick walls.

A hospital adaptive solution could be regarded as design that evolutes and develops based on climatic and ecological elements, as well as advances in science and technology. The design is approached as a living organism as if natural forces had shaped the architecture.

Modular solutions with simplified installation, and system integration that improves hospital performance should be applied to secure the flexibility and sustainability of the hospital building.

Natural ventilation and lighting are also good germicidal factors and prevent infection. There should be open space so that the natural air and light enters the rooms and corridors. Therefore, the solution of wards, middle corridors, loop corridors (square or round) or closed U-shaped wards that the foreign architects often design based entirely on the air conditioning system with disinfectant filtration systems cannot be completely trusted.

The strong points of sustainable hospital architecture design of the past must not be ignored. In reality, there are some very successful hospital design works sustained in Vietnam in the past that must be revised and followed. Notable samples are the Cho Ray Hospital and Grall hospital.

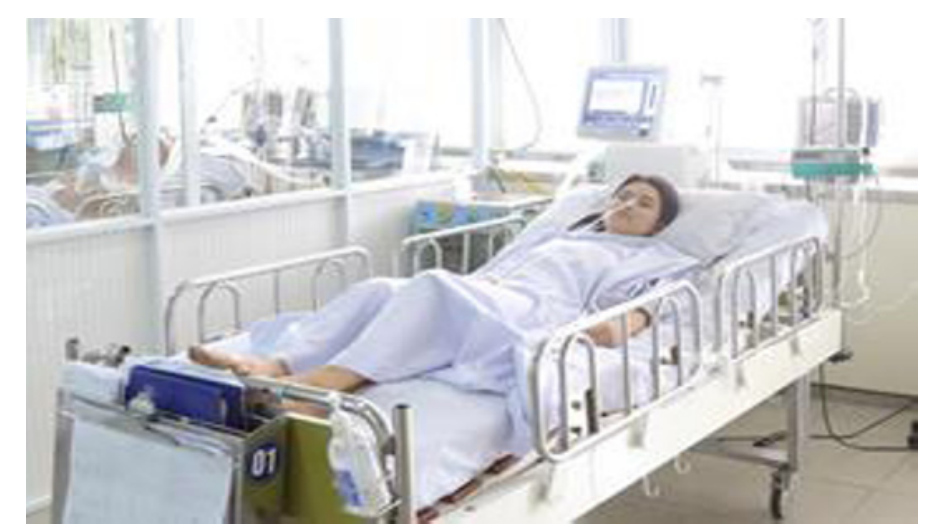

Fig.5. Grall Hospital, old part built in 1879 with open side corridors (Source Quang Chung) 


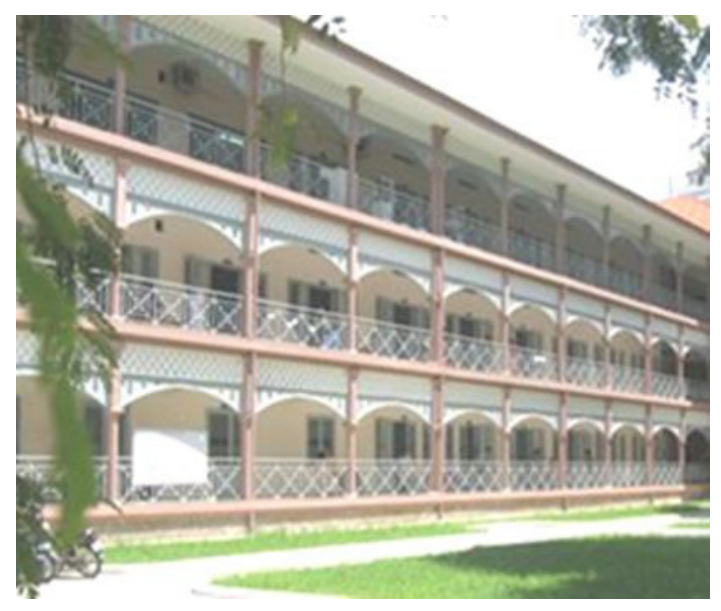

Fig.6. Cho Ray Hospital ICU room receiving natural light (Source:Khanh Trung)

Although built more than 30 years ago, Cho Ray Hospital still has many advantages: the inpatient zones are flooded with natural day light and ventilation through a wide side corridor, it saves energy efficiently and serves well for the recovery of patients' health. The operation zone is far from the elevator blocks and shafts. The walls are in metal panels which can be removed suitable with new demands of change in the new context without demolishing. Aluminium louvers are used instead of concrete solid types which can absorb heat and pathogens. It was no surprise when the Architect of Cho Ray hospital was Takeo Sato, one of the masters of Japanese modern architecture.

\section{Conclusion}

Many existing hospital builidings in Vietnam had been using the technology of more than 30 years ago and also have a certain number of disadvantages compared to this era. However some of the outstanding hospitals designed by Japanese, French and Vietnamese architects of the past era are more appropriate to the natural context of Vietnam than several new hospitals designed by current International architects, which depend on advanced technology systems but do not pay sufficient attention to climate change issues and the spark of global epidemics. Currently, the working chain: Investors - Architects - Engineers on structure and ME system of many design unit is deemed not to satisfy the establishment of a most effective sustainable project particularly the demand for the control of HAIs..

'A hospital sustainable design project of our era in Vietnam should be an adaptive solution carried out by a unit of multidisciplinary professional including architects, electrical engineers, equipment engineers, interior designers, construction contractors, equipment suppliers, project managers, financial managers, board of directors with hospital medical staff and not missing infection prevention experts. These experts in the above mentioned multidisciplinary unit must coordinate synchronously together in introducing and reviewing the most advanced technology solutions but flexible and appropriate with the Vietnam natural climate context, in line with requirements of infection prevention capacity to achive a most sustainable hospital project. 


\section{References}

1. S. Arora, An Evolutionary Architecture: Adapted, interactive, and effectively integrated design (2009).

2. J. Atkinson, Y. Chartier, L.Carmen, S. Pessoa, P. Jensen, Li Yuguo, S. Wing-Hong, Guidelines Natural Ventilation for Infection Control in Health-Care Settings.WHO Publication.

3. L. Buchbinder, et al., J Bacteriol. 42(5), 545-555 (1942).

4. Center for Health and the Global Environment, Harvard Medical School, 260 Longwood Avenue, Boston, MA 02115, USA . Climate change and emerging infectious diseases. Review of theories on infection control in hospital buildings. 3 (2001).

5. J.P. Chretien, A. Anyamba, J. Small, S. Britch, J.L. Sanchez, A.C. Halbach, C. Tucker, K.J. Linthicum, Global climate anomalies and potential infectious disease risks. 7 (2014-2015).

6. M. Cimons, Infectious Diseases Like It Hot: How Climate Change Helps Cholera and Salmonella Outbreaks (2015).

7. Clinical Educators Guide for the prevention and control of infection in healthcare. NHMRC, Commonwealth of Australia. 2010. Archived from the original on 2015-0405. Retrieved 2015-09-12.

8. N. N. Damani, Excerpt: Principles of Infection Control, a research project of the University of Cambridge in the UK, - Manual of Infection Control Procedures, 2nd Edition, Cambridge University Press 0521687012 (2003).

9. Gu,Yan \& Frazer, John H. Adaptive modelling of sustainable architecture design in entropy evolutionary Proceedings of The 12th Asia-Pacific Symposium on Intelligent and Evolutionary Systems, Clayton School of Information Technology, Monash University, University of Melbourne, Melbourne, Vic. (2008)

10. Hospital-acquired infections a threat to patients http://vietnamnews.vn/society/418150/ Update: November, 24/2017 - 09:49

11. U. Kazuyuki, The health impacts of climate change in Asia-Pacific. Asia-Pacific Human Development Report Background Papers Series 2012/16. (2012).

12. A.J. McMichael, A.Haines, R.Slooff, S. Kovats., Climate Change and Human Health. An Assessment by a Task Group on Behalf of the WHO the World Meteorological Organization and the United Nations Environment Programme. World Health Organization, Geneva, Switzerland (1996)

13. R. Kronenburg, Flexible architecture. (2019).

14. Sehulster, Lynne \& Y.W. Chinn Raymond (HICPAC) Guidelines for Environmental Infection Control in Health-Care Facilities.

15. Olamide Olusanya. Elegbede Adedayo, and Ogunseye Abiodun (2015) Department of Electrical / Electronic and Computer Engineering, College of Engineering, Bells University of Technology, P.M.B.1015, Ota, Ogun State. Nigeria IOSR Journal of Mobile Computing \& Application (IOSR-JMCA) e-ISSN: 2394-0050, P-ISSN: 23940042.Volume 2, Issue 1. (Mar. - Apr. 2015), PP 32-36 www.iosrjournals.org

16. PLoS One. 2018; 13(9): e0203600. Published online 2018 Sep 7. doi: 10.1371/journal.pone.0203600 PMCID: PMC6128614 PMID: 30192894

17. S.Hoffman, Employing E-Health: The Impact of Electronic Health Records on the Workplace (2010). 
18. Strategy and technology to prevent hospital-acquired infections: Lessons from SARS, Ebola, and MERS in Asia and West Africa.

19. R. Sprow, AIA . Planning Hospitals of the Future (2012).

20. Strong, DTG. FCIBSE, FEI Daylight benefits in healthcare buildings (2019).

21. J. Atkinson, et. Al., Natural ventilation for infection control in health-care settings. (2009).

22. Loo Lin Yong, et.al, Climate Change and Infectious Diseases: The Next 50 Years . 8, 47, 10 (2018). 